刘雪丽 ${ }^{1,2}$, 陈松长 ${ }^{1}$, 黄荷风 ${ }^{1,2}$, 徐晨明 ${ }^{1,2^{*}}$

1. 上海交通大学医学院附属国际和平妇幼保健院生殖遗传科, 上海 200030;

2. 上海市胚胎源性疾病重点实验室, 上海 200030

*联系人, E-mail: xuchenm@163.com

收稿日期：2019-12-30; 接受日期：2020-04-26; 网络版发表日期：2020-07-13

摘要 本研究以微阵列比较基因组杂交(array comparative genomic hybridization, aCGH)平台为参照, 旨在探究基 于下一代测序(next-generation sequencing, NGS)的胚胎植入前遗传学非整倍体检测(preimplantation genetic testing for aneuploidies, PGT-A)对高龄不育患者辅助生殖临床结局的影响. 本文回顾性分析了上海交通大学医学院附属 国际和平妇幼保健院2016年6月至2019年3月行PGT-A的104对夫妇的临床资料, 共纳入125个移植周期, 其中正常 胚胎移植周期为 107 个, 嵌合体胚胎移植周期为 18 个. 主要结果指标包括正常囊肧检出率和嵌合体囊胚检出率, 以 及临床妊娠率、流产率和活产率. 研究结果表明, 与 $\mathrm{aCGH}$ 平台相比, NGS的嵌合胚胎的检出率显著提高, 且部分 嵌合体胚胎移植获得了健康胎儿. 对于移植检测结果为正常的胚胎, 基于NGS的PGT-A和基于 aCGH的PGT-A对 高龄女性辅助生殖的临床妊娠率、流产率和活产率的影响无显著差异.

关键词高龄, 肧胎植入前遗传学非整倍体检测, 下一代测序, 微阵列比较基因组杂交

随着“全面二孩”政策的开放和现代女性生育年龄 的推迟, 高龄备孕女性的比重显著增加, 高龄女性的生 殖问题和改善高龄女性的生殖结局已成为生殖医学领 域关注的焦点. 非整倍体是人类胚胎最常见的染色体 异常, 是导致高龄女性体外受精(in vitro fertilization, IVF)中胚胎种植失败和流产以及胎儿出生缺陷的主要 原因, 并且随着女方年龄的增长, 其发生率显著增加 ${ }^{[1]}$. 因此, 选择整倍体胚胎进行移植有望提高体外受精-胚 胎移植的成功率 ${ }^{[2]}$.

肧胎植入前遗传学非整倍体篮查(preimplantation genetic testing for aneuploidies, PGT-A)是对行IVF的胚 胎进行染色体非整倍性检测，选择具有发育潜能的整 倍体胚胎移植, 以提高临床妊娠率、降低早期流产 率 $^{[3]}$. 传统应用于女性高龄(advanced maternal age, AMA)的PGT-A的荧光原位杂交(fluorescent in situ hybridization, FISH)技术仅能对胚胎的少数几条染色体 进行分析, 并不能提高临床妊娠率和胚胎终止率, 部 分文献报道其反而会降低活产率 ${ }^{[4]}$. 下一代测序(nextgeneration sequencing, NGS)和微阵列技术为代表的分 子诊断技术推动PGT技术实现了全基因组范围的染色

引用格式: 刘雪丽, 陈松长, 黄荷风, 等. 下一代测序技术在高龄女性的胚胎植入前非整倍体遗传学检测中的应用. 中国科学: 生命科学, 2020, 50: 866-873 Liu X L, Chen S C, Huang H F, et al. Application of preimplantation genetic testing for aneuploidies with next-generation sequencing in women with advanced maternal age (in Chinese). Sci Sin Vitae, 2020, 50: 866-873, doi: 10.1360/SSV-2019-0303 
体检测, 标志着PGT-A 新纪元的开始. 本研究拟通过比 较基于NGS和微阵列比较基因组杂交(array comparative genomic hybridization, aCGH)技术的PGT-A对高 龄不育患者的助孕结局，来探究NGS技术在胚胎植入 前遗传学篎查中的应用价值, 旨在帮助高龄女性做出 最佳生殖决策.

\section{1 资料与方法}

\section{1 研究对象}

本文回顾性研究了2016年6月至2019年3月于上海 交通大学医学院附属国际和平妇幼保健院行PGT-A的 104 对夫妇的助孕资料. 其中, 有74对夫妇采用下一代 测序技术(NGS组)，30对夫妇采用微阵列比较基因组 杂交技术( $\mathrm{aCGH}$ 组，为对照组), 进行囊胚活检和染色 体非整倍体检测. 纳入标准: 根据2019年《中国高龄不 孕女性辅助生殖临床实践指南》推荐意见 16 和欧洲人 类生殖和胚胎学会指南, 选择女方年龄 $\geq 36$ 岁 $^{[5,6]}$, 夫妻 双方染色体核型正常, 至少有一个可移植囊胚. 排除标 准: 合并严重输卵管积水或内分泌疾病. 所有患者均签 署知情同意书.

\section{2 促排卵及胚胎活检}

根据年龄、卵巢储备功能等选用黄体期长方案、 卵泡期超长方案、拮抗剂方案或微刺激等方案进行促 排卵. 扳机后35 36 h 取卵. MII卵采用卵胞浆内单精子 注射进行体外受精, 于采卵后第5 6天行胚胎评分后取 5 10个囊胚滋养层细胞活检. 利用SurePlex试剂盒(IIlumina，美国)对活检细胞进行全基因组扩增，VeriSeq ${ }^{\mathrm{TM}}$ PGS试剂盒(Illumina)进行文库制备. 2016年6月 至2017年8月主要使用 $\mathrm{aCGH}$ 平台检测，过渡期间主要 根据技术人员对平台的熟练程度随机使用 NGS或 $\mathrm{aCGH}$ 平台, 2018年2月后全部使用 NGS平台检测. 活检 后的胚胎行玻璃化冷冻, 于液氮中冻存.

\section{3 胚胎移植}

所有病例均采用冷冻-复苏周期的单囊胚移植. 其 中NGS组共 89 个移植周期, aCGH组共 36 个移植周期. 胚胎移植优先考虑正常整倍体且评分级别高的胚胎, 对于没有正常整倍体胚胎的患者建议重启促排卵周 期, 如果仍然没有正常整倍体胚胎可用, 在充分遗传咨
询后选择移植低风险的二倍体/非整倍体嵌合胚胎. 本 研究选择嵌合比例 20\% 50\%的胚胎用于移植，超过 $50 \%$ 的嵌合胚胎则不移植. 本研究共移植嵌合体胚胎 18例, 其中 aCGH组移植3例, NGS组移植 15 例, 所有嵌 合体移植的妊娠病例均进行羊水穿刺和细胞遗传学产 前诊断.

\section{4 观察指标}

主要观察指标包括临床妊娠率、流产率和活产 率. 生化妊娠定义为胚胎移植后出现血清促线毛膜激 素水平大于 $10 \mathrm{mIU} / \mathrm{mL}$ 后进入下个月经周期; 临床妊 娠定义为胚胎移植后28 30天通过阴道子宫超声发现 孕囊, 临床妊娠率 $(\%)=$ 每组临床妊娠数/每组移植周期 数; 流产率 $(\%)=$ 每组流产例数 $/$ 每组临床妊娠例数; 活 产率 $(\%)=$ 每组活产例数 $/$ 每组临床妊娠例数. 次要观察 指标包括正常、异常囊胚检出率和嵌合体囊胚检 出率.

\section{5 统计学方法}

采用SPSS 25软件进行统计分析. 计量资料以均数 土标准差表示, 计数资料以率 $(\%)$ 表示. 计量资料采用 $t$ 检验，计数资料采用卡方检验或Fisher确切概率法. $P<0.05$ 表示差异有统计学意义.

\section{2 结果}

NGS和aCGH两组患者的基本信息见表 1 . 夫妻双 方年龄、原发和继发不孕比例无显著性差异.

促排卵、胚胎体外培养及胚胎活检情况见表 2 . NGS组共获卵 815 个，其中成熟卵为 634 个，成熟率为 $77.8 \% ， 531$ 个胚胎正常受精，正常受精率为 $83.8 \%$ ，卵 裂 517 个, 卵裂率为 $97.4 \%$, 形成囊胚 215 个, 囊胚形成率 为 $41.6 \%$. aCGH组共获卵 298 个，其中成熟卵为 244 个, 成熟率为 $81.9 \% ， 205$ 个胚胎正常受精，正常受精率为 $84.0 \%$, 卵裂 200 个, 卵裂率为 $97.6 \%$, 形成囊胚 85 个, 囊 胚形成率为 $42.5 \%$. 两组患者的获卵数、MII卵数、两 原核 (2 pronuclear, $2 \mathrm{PN})$ 数、卵裂数和囊胚形成数均无 统计学差异.

两组患者活检囊胚总数无统计学差异. 图1选取了 NGS和aCGH检测结果一例有代表性的图示. NGS组和 $\mathrm{aCGH}$ 组在正常、异常和嵌合囊胚检出率上有统计学 
表 1 两组病例的基本临床资料

Table 1 Comparison of patients baseline between two groups

\begin{tabular}{cccc}
\hline & NGS组 & aCGH组 & $P$ 值 \\
\hline 病例数 $(n)$ & 74 & 30 & - \\
女方年龄(岁) & $38.03 \pm 2.16$ & $38.33 \pm 2.64$ & 0.541 \\
男方年龄(岁) & $39.85 \pm 5.11$ & $41.94 \pm 5.67$ & 0.071 \\
原发不孕数(\%) & $15(20.27)$ & $5(16.67)$ & 0.392 \\
继发不孕数(\%) & $59(79.73)$ & $25(83.33)$ & \\
\hline
\end{tabular}

表 2 两组病例的促排卵和胚胎培养及活检结果

Table 2 Comparison of embryologic outcomes and biopsy results between two groups

\begin{tabular}{cccc}
\hline & NGS组 $(n=74)$ & aCGH组 $(n=30)$ & $P$ 值 \\
\hline 获卵数 $(n)$ & $11.01 \pm 6.71$ & $9.93 \pm 6.51$ & 0.985 \\
MII卯数 $(n)$ & $8.57 \pm 5.21$ & $8.13 \pm 5.15$ & 0.991 \\
2PN数 $(n)$ & $7.18 \pm 4.60$ & $6.83 \pm 3.97$ & 0.968 \\
卵裂数 $(n)$ & $6.99 \pm 4.45$ & $6.67 \pm 3.99$ & 0.858 \\
囊胚形成数 $(n)$ & $2.77 \pm 1.79$ & $2.70 \pm 1.74$ & 0.858 \\
活检囊胚数 $(n)$ & $2.70 \pm 1.76$ & $2.07 \pm 1.55$ & 0.606 \\
正常囊胚率 $(\%)$ & $45.0(90 / 200)$ & $62.9(39 / 62)$ & $0.037^{\mathrm{a})}$ \\
异常囊胚率 $(\%)$ & $43.0(86 / 200)$ & $32.3(20 / 62)$ & \\
囊胚嵌合体率 $(\%)$ & $12.0(24 / 200)$ & $4.8(3 / 62)$ & \\
\hline
\end{tabular}

a) Fisher确切概率法

\section{差异 $(P<0.05)$.}

本研究总共有 107 个正常胚胎移植周期, 其妊娠结 局见表3. NGS组相对于 $\mathrm{aCGH}$ 组的临床妊娠率和活产 率升高, 流产率也升高, 但差异均无统计学意义. 在 18 个嵌合体移植胚胎中，共发现28处嵌合型染色体非整 倍体(表4)。其中，aCGH组发现了1处嵌合片段性非整 倍体, 4处嵌合全染色体非整倍体; NGS组发现了2处嵌 合片段性非整倍体，21处嵌合全染色体非整倍体. $\mathrm{aCGH}$ 组移植 3 例, 生化妊娠 1 例, 2例未妊娠; NGS组移 植 15 例, 生化妊娠 1 例, 临床妊娠3例且全部持续妊娠至 足月分婏, 其产前诊断结果均正常.

\section{3 讨论}

多项研究表明, 随着母亲孕龄的升高, 卵子发生过 程中的减数分裂异常或受精后有丝分裂异常的概率明 显增高，胚胎全染色体缺失/增加的机会也随之增 高 ${ }^{[2,7]}$, 发生率从女性20岁左右的 $25 \%$ 增加到 40 岁以上
的 $65 \%{ }^{[8,9]}$. 但有关女性年龄与胚胎发生片段性染色体 缺失/增加的风险是否相关尚无定论, 还有待更多大样 本研究的确定. Scott团队 ${ }^{[9]}$ 对不同年龄女性共 15169 个 囊胚的活检结果进行分析, 发现26 30岁女性非整倍体 发生率最低, 而超过35岁后非整倍体胚胎比例明显增 加. 胚胎染色体非整倍体是高龄妇女助孕失败和自然 流产的重要因素, 目前女性高龄是PGT-A较为公认的 适应证之一. Rubio等人 ${ }^{[10]}$ 的研究表明, 采用PGT-A篮 选出正常的整倍体胚胎移植, 不仅能显著提高高龄不 育患者首次胚胎移植的临床结局，还能显著降低流产 率并缩短妊娠时间.

NGS目前作为主流的高通量测序技术，可检测出 染色体倒位、平衡易位或罗伯逊易位导致的胚胎染色 体拷贝数异常及所有染色体的非整倍体异常, 并且在 检测速度和效率、灵敏度和准确度上均有明显的提 升, 可检测低至 $4 \mathrm{Mb}$ 左右长度的楛贝数变异和低至 $20 \%$ 的嵌合性异常 ${ }^{[1113]}$. 嵌合体胚胎由两种或以上的 细胞系构成, 是独立于整倍体胚胎和非整倍体胚胎的 

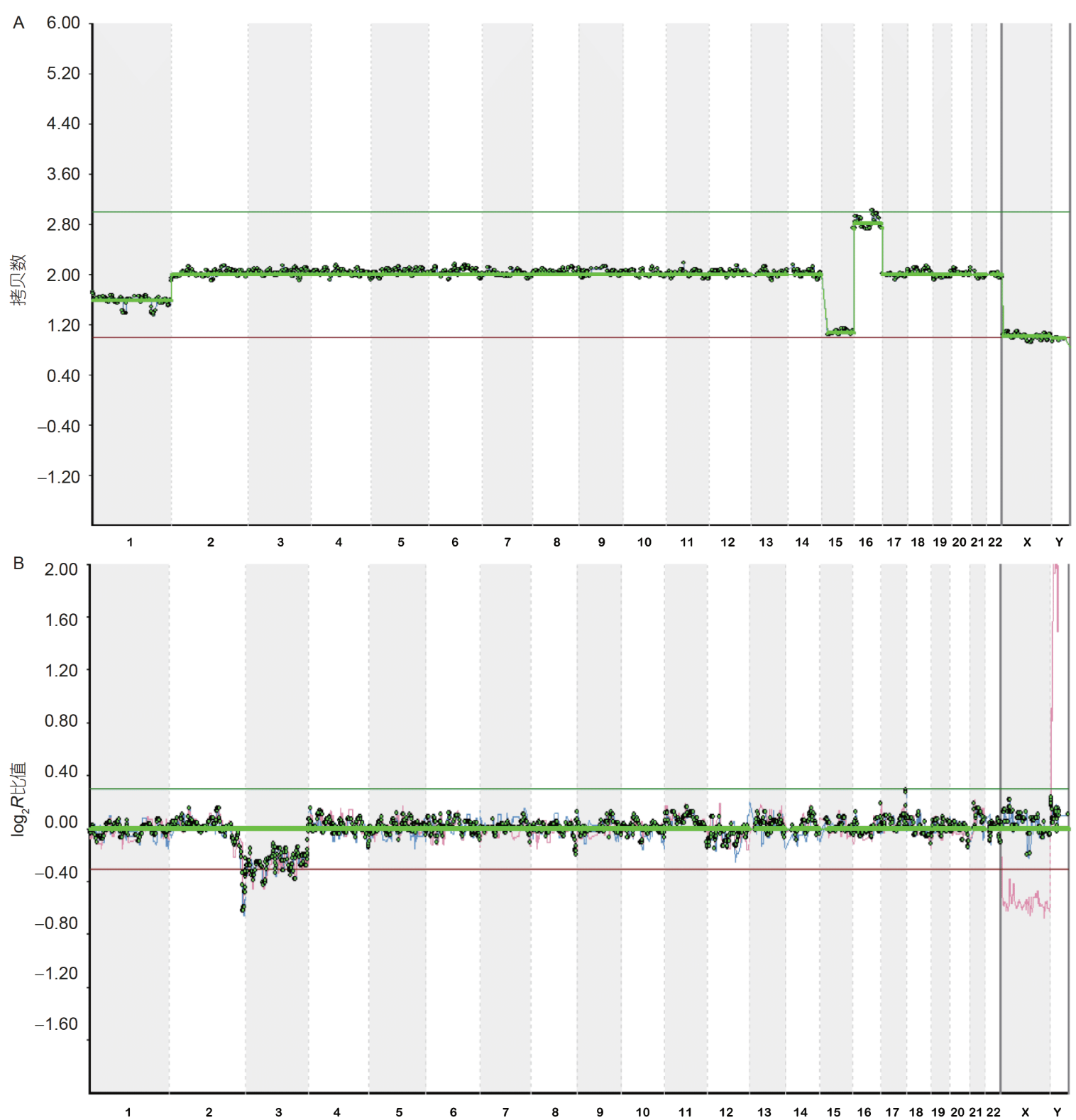

图 $1 \mathrm{NGS}$ 和 $\mathrm{aCGH}$ 检测的代表性图例. A: NGS检测结果, 胚胎核型为 $46, \mathrm{XX},-15,+16 / 45, \mathrm{XX},-1,-15,+16,1$ 号染色体缺失 的嵌合比例为 $30 \%$; B: aCGH检测结果, 胚胎核型为 $46, \mathrm{XX} / 45, \mathrm{XX},-3,3$ 号染色体缺失的嵌合比例为 $50 \%$ (网络版彩图)

Figure 1 Representative profiles detected by NGS and aCGH. A: Detected by NGS. Its karyotype was $46, \mathrm{XX},-15,+16 / 45, \mathrm{XX},-1,-15,+16$, and it revealed a $30 \%$ mosaicism of chromosome 1 . B: Detected by aCGH. Its karyotype was $46, \mathrm{XX} / 45, \mathrm{XX},-3$, and it revealed a $50 \%$ mosaicism of chromosome 3 (color online)

第三种结果. 自嵌合体胚胎被移植并诞下健康胎儿被 报道以来，嵌合体胚胎移植的临床结局和应用策略受 到广泛关注 ${ }^{[14 ~ 16]}$. 虽然在这些基于小样本研究报道中, 嵌合体的检出率、嵌合体胚胎移植对临床结局的影响 存在差异，但相关研究均表明，与整倍体胚胎移植相 比，嵌合体胚胎植入率更低，流产风险也明显升高 ${ }^{[14]}$.
目前达成的共识是, 在缺少整倍体胚胎, 且无条件再次 启动取卵周期的情况下，和患者经过充分的遗传咨询 和沟通，告知潜在问题，患者知情同意后可考虑选择 部分类型的嵌合体胚胎移植 ${ }^{[17]}$.

与NGS相比，aCGH的检测流程较为繁琐，对嵌合 体胚胎检测的敏感度也次于NGS，仅能准确检出非整 
表 3 两组病例正常囊胚移植周期的妊娠结局

Table 3 Comparison of the clinical outcomes after transferring normal embryo between two groups

\begin{tabular}{cccc}
\hline & NGS组 & aCGH组 & $P$ 值 \\
\hline 移植周期数 $(n)$ & 74 & 33 & - \\
临床妊娠率(\%) & $41.9(31 / 74)$ & $30.3(10 / 33)$ & $0.225^{\mathrm{a})}$ \\
生化妊娠率(\%) & $5.4(4 / 74)$ & $3.0(1 / 33)$ & $0.591^{\mathrm{b})}$ \\
流产率(\%) & $9.7(3 / 31)$ & $0.0(0 / 10)$ & $0.506^{\mathrm{b})}$ \\
活产率(\%) & $37.8(28 / 74)$ & $30.3(10 / 33)$ & $0.452^{\mathrm{a})}$ \\
\hline
\end{tabular}

a) 方差分析; b) Fisher确切概率法

表 4 两组病例嵌合体胚胎移植周期的妊娠结局

Table 4 Comparison of the clinical outcomes after transferring mosaic embryo between two groups

\begin{tabular}{|c|c|c|c|c|c|}
\hline 女性年龄(岁) & 病例编号-胚胎编号 & 检测平台 & 胚胎检测结果 & 嵌合比例 & 临床结局 \\
\hline \multirow{2}{*}{40} & \multirow{2}{*}{$21-3$} & \multirow{2}{*}{$\mathrm{aCGH}$} & $1 \mathrm{p} 11.2-1 \mathrm{q} 43$ 缺失 & $50 \%$ & \multirow{2}{*}{ 未妊娠 } \\
\hline & & & 10 号染色体缺失 & $40 \%$ & \\
\hline \multirow{2}{*}{38} & \multirow{2}{*}{$24-7$} & \multirow{2}{*}{$\mathrm{aCGH}$} & 19号染色体缺失 & $40 \%$ & \multirow{2}{*}{ 生化妊娠 } \\
\hline & & & 20 号染色体缺失 & $40 \%$ & \\
\hline 36 & $29-3$ & $\mathrm{aCGH}$ & 3号染色体缺失 & $40 \%$ & 未妊娠 \\
\hline \multirow{2}{*}{39} & \multirow{2}{*}{$49-3$} & \multirow{2}{*}{ NGS } & 8 号染色体重复 & $20 \%$ & \multirow{2}{*}{ 活产 } \\
\hline & & & 12 号染色体缺失 & $20 \%$ & \\
\hline 39 & $53-4$ & NGS & 5 号染色体重复 & $30 \%$ & 未妊娠 \\
\hline \multirow{2}{*}{41} & \multirow{2}{*}{$59-1$} & \multirow{2}{*}{ NGS } & 2号染色体重复 & $20 \%$ & \multirow{2}{*}{ 未妊娠 } \\
\hline & & & 12 号染色体缺失 & $20 \%$ & \\
\hline 37 & $75-2$ & NGS & 21号染色体缺失 & $30 \%$ & 未妊娠 \\
\hline \multirow{2}{*}{37} & \multirow{2}{*}{$77-3$} & \multirow{2}{*}{ NGS } & 2q22.1-2q37.1重复 & $40 \%$ & \multirow{2}{*}{ 活产 } \\
\hline & & & 16 号染色体缺失 & $30 \%$ & \\
\hline 40 & $79-5$ & NGS & 3号染色体重复 & $20 \%$ & 未妊娠 \\
\hline \multirow{2}{*}{39} & \multirow{2}{*}{$80-2$} & \multirow{2}{*}{ NGS } & 11 号染色体重复 & $20 \%$ & \multirow{2}{*}{ 未妊娠 } \\
\hline & & & 17号染色体重复 & $30 \%$ & \\
\hline 39 & $87-7$ & NGS & 1 号染色体缺失 & $20 \%$ & 未妊娠 \\
\hline 36 & $88-6$ & NGS & 22号染色体重复 & $20 \%$ & 未妊娠 \\
\hline 36 & $97-1$ & NGS & 19号染色体重复 & $30 \%$ & 活产 \\
\hline \multirow{2}{*}{37} & \multirow{2}{*}{$98-2$} & \multirow{2}{*}{ NGS } & 11号染色体重复 & $20 \%$ & \multirow{2}{*}{ 未妊娠 } \\
\hline & & & 19号染色体重复 & $20 \%$ & \\
\hline 38 & $99-2$ & NGS & Xq25-Xq28缺失 & $40 \%$ & 未妊娠 \\
\hline \multirow{2}{*}{37} & \multirow{2}{*}{$102-2$} & \multirow{2}{*}{ NGS } & 9号染色体缺失 & $30 \%$ & \multirow{2}{*}{ 生化妊娠 } \\
\hline & & & 19号染色体重复 & $30 \%$ & \\
\hline 36 & $103-2$ & NGS & 20 号染色体缺失 & $20 \%$ & 未妊娠 \\
\hline & & & 4号染色体重复 & $20 \%$ & \\
\hline 37 & $104-3$ & NGS & 7号染色体重复 & $20 \%$ & 未妊娠 \\
\hline & & & 14号染色体重复 & $20 \%$ & \\
\hline
\end{tabular}


倍体细胞占 $40 \%$ 60\%范围的胚胎 ${ }^{[18]}$. 这就有可能将有 潜能发育成健康胎儿的嵌合体胚胎被沴断成异常肧 胎, 导致周期取消率高和胚胎浪费, 或者嵌合胚胎被错 估为正常的整倍体胚胎, 导致潜在的妊娠风险. 本研究 结果显示, 与 $\mathrm{aCGH}$ 组相比, NGS组正常胚胎检出率降 低, 嵌合体胚胎检出增多, 推测临床上相当一部分使用 $\mathrm{aCGH}$ 评估为核型正常的胚胎实际上为嵌合体胚胎, 因 此NGS检测在一定程度上可减少 $\mathrm{aCGH}$ 造成无效移植 周期. 本研究的移植嵌合体胚胎的例数少, 因此还需要 进一步积累资料探究嵌合体胚胎的妊娠结局.

此外，高通量测序技术作为第二代PGT-A(相对于 基于FISH的第一代PGT-A)的引领技术显著提高了 IVF 高龄不孕患者的妊娠结局 ${ }^{[19-22]}$. 本研究回顾性分析了 胚胎植入前遗传学筛查中作为新兴的遗传检测技术 $\mathrm{NGS}$ 和准确度高度认可的 $\mathrm{aCGH}$ 两个高通量测序平台
对高龄不孕患者的助孕结局的影响，与 $\mathrm{aCGH}$ 组相比， NGS组的临床妊娠率和活产率均有提高, 但差异没有 显著性意义. 但相对于高龄不孕患者仅行IVF的流产 率 $16.2 \% \sim 44.88 \%{ }^{[23 \sim 25]}$ ，本研究基于 $\mathrm{NGS}$ 或 $\mathrm{aCGH}$ 的第 二代PGT-A技术的流产率均明显降低. 基于本研究结 果，推测在整体高龄范围上基于NGS的PGT-A相对基 于 $\mathrm{aCGH}$ 的PGT-A对提升妊娠结局没有优势, 但由于 NGS检测准确度的提升, 如果对高龄范围进行更细致 的分层也许可以找到明显受益于 NGS技术的年龄阶 层, 因此还需扩大样本量进一步研究.

本次研究为回顾性研究，两种技术的应用时间不 完全同步，存在一定的偏倚，具有一定局限性. 总之, NGS技术显著提高了胚胎检测的准确度和灵敏度，基 于NGS与基于 $\mathrm{aCGH}$ 的PGT-A对行正常整倍体胚胎移 植的整体高龄女性妊娠结局的影响无显著差异.

\section{参考文献}

1 Simon A L, Kiehl M, Fischer E, et al. Pregnancy outcomes from more than 1,800 in vitro fertilization cycles with the use of 24-chromosome single-nucleotide polymorphism-based preimplantation genetic testing for aneuploidy. Fertil Steril, 2018, 110: 113-121

2 Babariya D, Fragouli E, Alfarawati S, et al. The incidence and origin of segmental aneuploidy in human oocytes and preimplantation embryos. Hum Reprod, 2017, 32: 2549-2560

3 Verpoest W, Staessen C, Bossuyt P M, et al. Preimplantation genetic testing for aneuploidy by microarray analysis of polar bodies in advanced maternal age: a randomized clinical trial. Hum Reprod, 2018, 33: 1767-1776

4 Mastenbroek S, Twisk M, van der Veen F, et al. Preimplantation genetic screening: a systematic review and meta-analysis of RCTs. Hum Reprod Update, 2011, 17: 454-466

5 Chinese Society of Reproductive Medicine (CSRM). Chinese practice guideline on the assisted reproductive technology (ART) strategies for women with advanced age (in Chinese). Chin J Evid-Based Med, 2019, 19: 253-270 [中华医学会生殖医学分会. 中国高龄不孕女性辅助生殖 临床实践指南. 中国循证医学杂志, 2019, 19: 253-270]

6 Harton G, Braude P, Lashwood A, et al. ESHRE PGD consortium best practice guidelines for organization of a PGD centre for PGD/ preimplantation genetic screening. Hum Reprod, 2011, 26: 14-24

7 Demko Z P, Simon A L, McCoy R C, et al. Effects of maternal age on euploidy rates in a large cohort of embryos analyzed with 24-chromosome single-nucleotide polymorphism-based preimplantation genetic screening. Fertil Steril, 2016, 105: 1307-1313

8 Munné S, Chen S, Colls P, et al. Maternal age, morphology, development and chromosome abnormalities in over 6000 cleavage-stage embryos. Reprod Biomed Online, 2007, 14: 628-634

9 Franasiak J M, Forman E J, Hong K H, et al. The nature of aneuploidy with increasing age of the female partner: a review of 15,169 consecutive trophectoderm biopsies evaluated with comprehensive chromosomal screening. Fertil Steril, 2014, 101: 656-663.e1

10 Rubio C, Bellver J, Rodrigo L, et al. In vitro fertilization with preimplantation genetic diagnosis for aneuploidies in advanced maternal age: a randomized, controlled study. Fertil Steril, 2017, 107: 1122-1129

11 Vendrell X, Fernández-Pedrosa V, Triviño J C, et al. New protocol based on massive parallel sequencing for aneuploidy screening of preimplantation human embryos. Syst Biol Reprod Med, 2017, 63: 162-178

12 Zhang W, Liu Y, Wang L, et al. Clinical application of next-generation sequencing in preimplantation genetic diagnosis cycles for Robertsonian and reciprocal translocations. J Assist Reprod Genet, 2016, 33: 899-906

13 Fiorentino F, Biricik A, Bono S, et al. Development and validation of a next-generation sequencing-based protocol for 24-chromosome 
aneuploidy screening of embryos. Fertil Steril, 2014, 101: 1375-1382.e2

14 Fragouli E, Alfarawati S, Spath K, et al. Analysis of implantation and ongoing pregnancy rates following the transfer of mosaic diploid-aneuploid blastocysts. Hum Genet, 2017, 136: 805-819

15 Greco E, Minasi M G, Fiorentino F. Healthy babies after intrauterine transfer of mosaic aneuploid blastocysts. N Engl J Med, 2015, 373: 20892090

16 Zhang L, Wei D, Zhu Y, et al. Rates of live birth after mosaic embryo transfer compared with euploid embryo transfer. J Assist Reprod Genet, 2019, 36: 165-172

17 Grkovic S, Traversa M, Bonifacio M, et al. Challenges in interpreting the relevance of segmental mosaicism detected by NGS. Reprod Biomed Online, 2018, 36: e10-e11

18 Munné S, Wells D. Detection of mosaicism at blastocyst stage with the use of high-resolution next-generation sequencing. Fertil Steril, 2017, 107: 1085-1091

19 Harton G L, Munné S, Surrey M, et al. Diminished effect of maternal age on implantation after preimplantation genetic diagnosis with array comparative genomic hybridization. Fertil Steril, 2013, 100: 1695-1703

20 Munné S, Cohen J. Advanced maternal age patients benefit from preimplantation genetic diagnosis of aneuploidy. Fertil Steril, 2017, 107: 11451146

21 Sacchi L, Albani E, Cesana A, et al. Preimplantation genetic testing for aneuploidy improves clinical, gestational, and neonatal outcomes in advanced maternal age patients without compromising cumulative live-birth rate. J Assist Reprod Genet, 2019, 36: $2493-2504$

22 Bellver J, Bosch E, Espinós J J, et al. Second-generation preimplantation genetic testing for aneuploidy in assisted reproduction: a SWOT analysis. Reprod Biomed Online, 2019, 39: 905-915

23 Yang P Y, Ma C H, Chen L X, et al. Influence of decreasing the number of embryos transferred on pregnancy outcomes in advanced age women undergoing in vitro fertilization-embryo transfer (in Chinese). Chin J Reprod Contracep, 2018, 38: 831-836 [杨璞玉, 马彩虹, 陈立雪, 等. 减少 胚胎移植数量对高龄妇女体外受精-胚胎移植临床结局的影响. 中华生殖与避孕杂志, 2018, 38: 831-836]

24 Tannus S, Son W Y, Dahan M H. Elective single blastocyst transfer in advanced maternal age. J Assist Reprod Genet, 2017, 34: 741-748

Prevention CfDCa. 2016 Assisted Reproductive Technology National Summary Report. 2018 


\title{
Application of preimplantation genetic testing for aneuploidies with next-generation sequencing in women with advanced maternal age
}

\author{
LIU XueLi ${ }^{1,2}$, CHEN SongChang ${ }^{1}$, HUANG HeFeng ${ }^{1,2} \&$ XU ChenMing ${ }^{1,2}$ \\ 1 International Peace Maternity and Child Health Hospital, School of Medicine, Shanghai Jiao Tong University, Shanghai 200030, China; \\ 2 Shanghai Key Laboratory of Embryo Original Diseases, Shanghai 200030, China
}

This study aimed to explore the effects of next-generation sequencing (NGS) based preimplantation genetic testing for aneuploidies (PGT-A) on women with advanced maternal age compared with array comparative genomic hybridization (aCGH) based PGT-A. We retrospectively analyzed 125 frozen-thawed embryo transfers (FET) cycles from 104 couples, including 107 FET cycles with normal embryos and 18 FET cycles with mosaic embryos. The main outcomes included the detection rate of normal embryos, the detection rate of mosaic embryos, the clinical pregnancy rate, the miscarriage rate and live birth rate. The detection rate of mosaic embryos was significantly higher in NGS group compared with aCGH group. The transfer of a portion of mosaic embryos led to healthy births. For embryos with normal results, the clinical pregnancy rate, the miscarriage rate and live birth rate of NGS based PGT-A and aCGH based PGT-A were of no significant differences.

advanced maternal age, preimplantation genetic testing for aneuploidies, next-generation sequencing, array comparative genomic hybridization

doi: $10.1360 /$ SSV-2019-0303 